\title{
99mTc-Glu-c(RGDyK)-Bombesin SPECT Can Reduce Unnecessary Biopsy of Masses That Are BI-RADS Category 4 on Ultrasonography
}

\author{
Tiefeng Ji*1, Shi Gao*1, Zhaofei Liu ${ }^{2}$, Hua Xing ${ }^{3}$, Guoqing Zhao ${ }^{1}$, and Qingjie $\mathrm{Ma}^{1}$ \\ ${ }^{1}$ Department of Nuclear Medicine, China-Japan Union Hospital of Jilin University, Changchun, China; ${ }^{2}$ Medical Isotopes Research \\ Center, Peking University, Beijing, China; and ${ }^{3}$ Department of Breast Surgery, China-Japan Union Hospital of Jilin University, \\ Changchun, China
}

\begin{abstract}
Masses that, on ultrasonography, are category 4 according to the Breast Imaging Reporting and Data System (BI-RADS) represent possible malignancy, and a biopsy is recommended. This study explored the value of $99 \mathrm{mTc}$-Glu-c(RGDyK)-bombesin (99mTc-RGD-bombesin) in reducing unnecessary biopsy of these masses. Methods: Ninety women with a BI-RADS 4 mass on ultrasonography were enrolled in this study to undergo breast SPECT using 99mTc-RGD-bombesin. The images were independently interpreted using qualitative visual and semiquantitative analyses. The final diagnosis was based on histopathologic examination of surgically excised or percutaneous biopsy specimens. Fractions of the samples were immunohistochemically analyzed to evaluate expression of integrin $\alpha_{v} \beta_{3}$ and gastrinreleasing peptide receptor (GRPR). The receptor-positive group was further divided into 3 subgroups (GRPR ${ }^{+} / \alpha_{v} \beta_{3}{ }^{+}, \mathrm{GRPR}^{+} / \mathrm{a}_{\mathrm{v}} \beta_{3}{ }^{-}$, and $\mathrm{a}_{\mathrm{v}} \beta_{3}{ }^{+} / \mathrm{GRPR}^{-}$). Results: Ninety-four masses (22 malignant and 72 benign) were confirmed by histopathologic examination. On qualitative analysis, 20 of the malignant masses showed high ${ }^{99 m T c-R G D-}$ bombesin accumulation and 48 of the benign masses showed no 99mTc-RGD-bombesin accumulation. The optimal cutoff for qualitative analysis was a score of 2 . Semiquantitative analysis revealed that 20 of the malignant masses and 16 of the benign masses had a relatively high tumor-to-normal-tissue ratio $(T / N)$. The optimal cutoff was a $\mathrm{T} / \mathrm{N}$ of 2.26. The mean $\mathrm{T} / \mathrm{N}$ was higher for malignant masses than for benign masses ( $3.17 \pm 0.86$ vs. $1.89 \pm 0.71, P<0.05)$. T/Ns did not differ among the 3 subgroups $(P>0.05)$. The areas under the receiver-operating-characteristic curves for the qualitative and semiquantitative analyses were 0.788 and 0.865 , respectively, and the overall diagnostic performance did not significantly differ between these analyses $(P>0.05)$. Conclusion: 99mTc-RGD-bombesin SPECT can differentiate benign from malignant BI-RADS 4 masses with high specificity. Further study of the application of this test to clinical breast cancer appears warranted.
\end{abstract}

Key Words: 99mTc-RGD-bombesin; SPECT; GRPR; integrin $a_{v} \beta_{3}$; breast lesions

J Nucl Med 2016; 57:1196-1200

DOI: 10.2967/jnumed.115.168773

Received Oct. 27, 2015; revision accepted Feb. 23, 2016.

For correspondence or reprints contact: Qingjie Ma, Jilin University, No. 126 Xian Tai Ave., Changchun, Ji, China 130033.

E-mail: maqj@jlu.edu.cn

${ }^{*}$ Contributed equally to this work.

Published online Mar. 24, 2016.

COPYRIGHT (c) 2016 by the Society of Nuclear Medicine and Molecular Imaging, Inc.
B reast cancer is the most prevalent malignant disease among women worldwide (1,2). In the United States, an estimated 234,190 new cases of breast cancer were expected to be diagnosed in 2015 , and these were expected to account for $29 \%$ of all cancer diagnosed in women (3). As such, breast cancer has become a major health problem. Developments in imaging technology have significantly increased the 5-y survival rates of breast cancer patients and improved their quality of life, as reported by the American Cancer Society.

To standardize the process of reporting breast masses, the American College of Radiology first published its Breast Imaging Reporting and Data System (BI-RADS) in 1992. According to the 2013 edition of the ACR BI-RADS Atlas, malignant and benign masses are differentiated according to features such as margin, shape, orientation, lesion boundary, and blood flow (4). BI-RADS category 4 represents possibly malignant masses, for which biopsy is recommended (5). However, BI-RADS categorization is subjective. The results of BI-RADS 4 biopsy vary considerably, and the cancer-to-biopsy yields are relatively low (22\%-33\%) (6-9). Moreover, unnecessary biopsies are associated with negative consequences such as pain, fear, anxiety, direct financial expenses, and risk of complications (10-12). Therefore, a predictor for benign BI-RADS 4 masses would be beneficial.

Dual receptor-targeting imaging was recently developed and could minimize unnecessary biopsies. This technique is based on the ability of a tracer to simultaneously combine with 2 targets on the surface of breast cancer cells. Moreover, tracer uptake can be semiquantitatively estimated through the tumor-to-normal-tissue ratio $(\mathrm{T} / \mathrm{N})$. Thus, dual receptor-targeting imaging could complement conventional techniques and improve diagnostic performance (13).

In our previous study, we found that the dual-targeting tracer ${ }^{99 m}$ Tc-HYNIC-Glu-c(RGDyK)-bombesin ( ${ }^{99 m}$ Tc-RGD-bombesin) bound not only to integrin $\alpha_{\mathrm{v}} \beta_{3}$ but also to gastrin-releasing peptide receptor (GRPR) in breast cancer. In addition, the tracer exhibited excellent safety for breast imaging (14). Thus, the current study focused exclusively on patients with BI-RADS 4 masses to determine the utility of ${ }^{99} \mathrm{mTc}-\mathrm{RGD}$-bombesin SPECT in filtering out benign masses and reducing the number of unnecessary biopsies in this population.

\section{MATERIALS AND METHODS}

\section{Subjects}

Ninety women (mean age, $54 \pm 12 \mathrm{y}$; mean weight, $63 \pm 14 \mathrm{~kg}$ ) with a BI-RADS 4 solid breast mass found on ultrasonography were 
enrolled. The patients had no history of breast disease. The exclusion criteria included pregnancy and lactation. The final diagnosis was based on histopathologic examination of surgically excised or percutaneous biopsy specimens. The Ethics Committees of the China-Japan Union Hospital of Jilin University approved the study, and all subjects gave written informed consent.

\section{9mTC-RGD-Bombesin SPECT}

Radiolabeling and quality control of the ${ }^{99 \mathrm{~m}} \mathrm{Tc}-\mathrm{RGD}$-bombesin were performed as described previously $(14,15)$. Specifically, a single 11.1 MBq/kg intravenous bolus of ${ }^{99 m} \mathrm{Tc}-\mathrm{RGD}$-bombesin was injected and then followed by a $10-\mathrm{mL}$ saline flush (14). Four hours after administration of the tracer, a dual-head, large-field-of-view scintillation camera (Skylight; Philips Healthcare) equipped with a lowenergy, parallel-hole collimator was used to acquire the SPECT emission images while the patients lay supine with their arms elevated.

The images were acquired every $20 \mathrm{~s}$ at a $3^{\circ}$ angle in a circular orbit of $180^{\circ}$ per detector array using a $20 \%$ energy window centered on $140 \mathrm{keV}$ and a $128 \times 128$ matrix. Subsequently, the images were reoriented to obtain transaxial, coronal, and sagittal views.

All images were independently interpreted qualitatively and semiquantitatively by 2 experienced nuclear medicine physicians who were unaware of the clinical history and other test results of the patients. First, the interpreters visually classified the $99 \mathrm{~m}$ Tc-RGD-bombesin uptake as grade 1, no abnormal obvious uptake (no uptake greater than that in contralateral or peripheral normal breast tissue), grade 2 , mildly increased uptake (lower than or equal to that in the mediastinum), or grade 3, definite focus of increased uptake (greater than that in the mediastinum). Disagreements on the interpretation were resolved by consensus. Then, a region of interest that included the area of intense uptake was drawn manually. If no obvious uptake was seen, the region was drawn over the area that had been abnormal on ultrasound. A region of the same size was then drawn on the contralateral normal breast. T/Ns were obtained by comparison of the radioactive counts of the two regions.

\section{Immunohistochemistry of GRPR and $\boldsymbol{\alpha}_{\mathrm{v}} \boldsymbol{\beta}_{\mathbf{3}}$ Expression}

GRPR and $\alpha_{\mathrm{v}} \beta_{3}$ expression was analyzed by immunohistochemistry, as described previously with some modifications $(16,17)$. The abnormal tissues were snap-frozen, sectioned $(3 \mu \mathrm{m})$, fixed with ice-cold acetone, rinsed with phosphate-buffered saline, and blocked with $10 \%$ goat serum for $30 \mathrm{~min}$ at room temperature. The sections were incubated with 1:100 goat anti-GRPR antibody (Santa Cruz Biotechnology) or with 1:100 humanized antihuman integrin $\alpha_{\mathrm{v}} \beta_{3}$ antibody $(20 \mu \mathrm{g} / \mathrm{mL}$ ) (Abegrin; BD Biosciences) for $1 \mathrm{~h}$ at room temperature. Three senior pathologists who were unaware of the test results independently assessed the intensity of staining as 1 (none), 2 (weak), or 3 (strong) and the amount of stained cells as 1 (none), $2(<10 \%), 3(10 \%-50 \%), 4(50 \%-90 \%)$, or 5 (all). Tumors were considered positive if the summed scores for intensity and amount were more than 6-that is, strong staining in at least $10 \%$ of cells or weak staining in over half the tumor-cell population (18).

The receptor expression of the masses was classified as $\mathrm{P}$ (positive for at least one receptor) or $\mathrm{N}$ (negative) and then further divided into a p1 subgroup (positive for both receptors $\left[\mathrm{GRPR}^{+} / \alpha_{\mathrm{v}} \beta_{3}{ }^{+}\right]$), a $\mathrm{p} 2$ subgroup (positive for GRPR only [GRPR $\left.{ }^{+} / \alpha_{\mathrm{v}} \beta_{3}{ }^{-}\right]$), or a p3 subgroup (positive for $\alpha_{\mathrm{v}} \beta_{3}$ only $\left[\alpha_{\mathrm{v}} \beta_{3}{ }^{+} / \mathrm{GRPR}^{-}\right]$).

\section{Statistical Analysis}

Data are expressed as mean $\pm \mathrm{SD}$. The Student $t$ test for unpaired data was used to compare differences in $\mathrm{T} / \mathrm{N}$ between malignant and benign masses and between the $\mathrm{P}$ and $\mathrm{N}$ groups. ANOVA was used to assess the significance of differences in T/Ns among the subgroups.
The sensitivity, specificity, accuracy, positive predictive value, negative predictive value, and accuracy of the SPECT qualitative and semiquantitative analyses were calculated according to the final diagnosis. Receiver-operating-characteristic analyses were performed to determine the optimal cutoffs for each measurement parameter and to evaluate the diagnostic performance of each measurement method. A $P$ value of less than 0.05 was considered to indicate a significant difference.

\section{RESULTS}

All patients underwent percutaneous biopsy within $7 \mathrm{~d}$ after SPECT, and 22 patients subsequently underwent surgery. In total, 94 masses were confirmed by histopathologic examination. Twenty-two malignant and 72 benign masses were identified. Their characteristics and measured parameters are presented in Table 1.

In the qualitative visual analysis, 20 of the malignant masses exhibited high ${ }^{99} \mathrm{~m}$ Tc-RGD-bombesin accumulation. The other 2 malignant masses did not take up tracer and could not be identified (a ductal carcinoma in situ with a diameter of $0.6 \mathrm{~cm}$ and an invasive ductal carcinoma with a diameter of $1.7 \mathrm{~cm}$ ). In contrast, 48 of the benign masses exhibited no uptake and the other 24 exhibited relatively high uptake. The optimal cutoff was a score of 2. A representative true-positive case and a representative falsenegative case are shown in Figures 1 and 2, respectively.

In the semiquantitative analysis, 20 malignant masses and 16 benign masses had relatively high T/Ns, whereas the remaining 2 malignant and 56 benign masses had relatively low T/Ns. The optimal $\mathrm{T} / \mathrm{N}$ cutoff was 2.26 . The mean $\mathrm{T} / \mathrm{N}$ for the malignant masses was $3.17 \pm 0.86$, which was significantly higher than that for the benign masses $(1.89 \pm 0.71, P<0.05$, Fig. 3$)$. The T/Ns differed significantly between the $\mathrm{P}$ and $\mathrm{N}$ groups (Fig. $4, P<0.05$ ) but not among the p1, p2, and p3 subgroups (Fig. 5, $P>0.05$ ).

TABLE 1

Mass Characteristics

\begin{tabular}{|c|c|}
\hline Characteristic & Number \\
\hline Malignant & 22 \\
\hline Invasive ductal carcinoma & 19 \\
\hline Ductal carcinoma in situ & 2 \\
\hline Invasive lobular carcinoma & 1 \\
\hline $\mathrm{GRPR}^{+} / \mathrm{a}_{\mathrm{v}} \beta_{3}{ }^{+}$ & 10 \\
\hline $\mathrm{GRPR}^{+} / \mathrm{a}_{\mathrm{v}}{\mathrm{\beta}_{3}^{-}}^{-}$ & 6 \\
\hline $\mathrm{GRPR}^{-} / \mathrm{a}_{\mathrm{v}} \beta_{3}{ }^{+}$ & 5 \\
\hline $\mathrm{GRPR}^{-} / \mathrm{a}_{\mathrm{v}} \beta_{3}^{-}$ & 1 \\
\hline Benign & 72 \\
\hline Fibroadenoma & 39 \\
\hline Adenosis & 29 \\
\hline Cyst & 3 \\
\hline Mammary tuberculosis & 1 \\
\hline $\mathrm{GRPR}^{+} / \mathrm{a}_{\mathrm{v}} \beta_{3}{ }^{+}$ & 6 \\
\hline $\mathrm{GRPR}^{+} / \mathrm{a}_{\mathrm{v}} \beta_{3}^{-}$ & 7 \\
\hline $\mathrm{GRPR}^{-} / \mathrm{a}_{\mathrm{v}} \beta_{3}{ }^{+}$ & 11 \\
\hline $\mathrm{GRPR}^{-} / \mathrm{a}_{\mathrm{v}} \beta_{3}^{-}$ & 48 \\
\hline
\end{tabular}



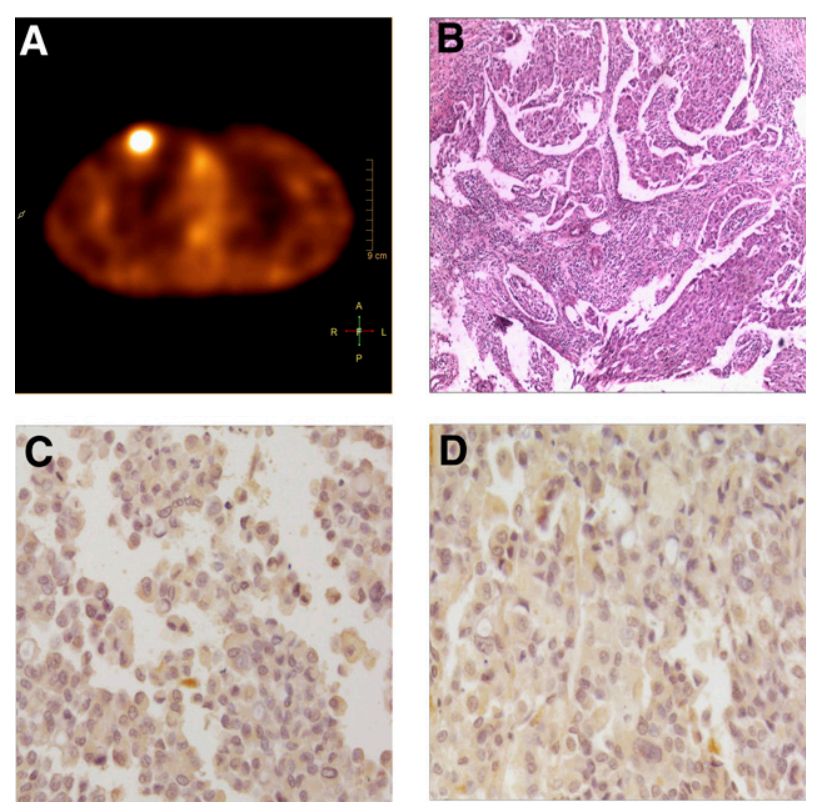

FIGURE 1. True-positive case. (A) High radiotracer uptake was observed in right breast. (B) Histopathologic staining indicated ductal carcinoma. Scale bar $=100 \mu \mathrm{m}$. (C and D) Immunohistochemistry revealed intense $\alpha_{v} \beta_{3}$ and GRPR expression both in tumor vessels and in tumor cells. Scale bar $=400 \mu \mathrm{m}$.

The sensitivity, specificity, accuracy, positive predictive value, and negative predictive value of the qualitative and semiquantitative analyses are presented in Table 2 . The areas under the empiric receiver-operating-characteristic curves for the qualitative and semiquantitative analyses were 0.788 and 0.865 , respectively,
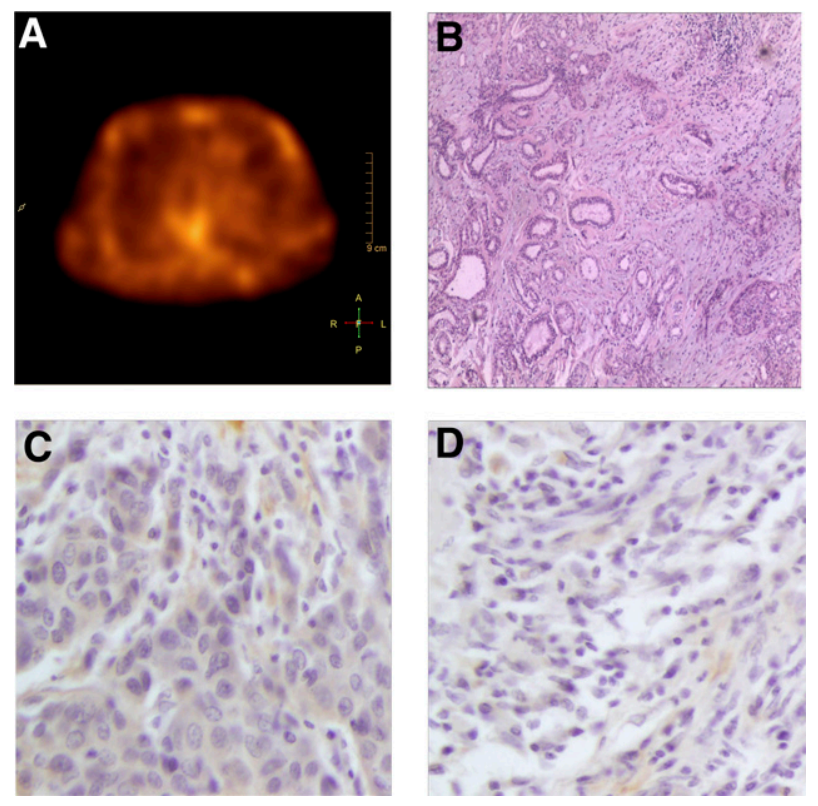

FIGURE 2. False-negative case. (A) No uptake was observed in either breast. (B) Histopathologic staining indicated invasive ductal carcinoma in left breast. Scale bar $=100 \mu \mathrm{m}$. (C and D) Immunohistochemistry revealed minimal $a_{v} \beta_{3}$ and GRPR expression in tumor vessels and tumor cells. Scale bar $=400 \mu \mathrm{m}$. and their overall diagnostic performance did not significantly differ $(P>0.05$, Fig. 6).

\section{DISCUSSION}

Compared with conventional modalities, nuclear receptor imaging, which is based on the high expression of receptors on cancer cells or in cancer microenvironments, has previously been demonstrated to have excellent diagnostic performance in the evaluation of breast masses (19-21). Nevertheless, patients who have already received a final diagnosis by conventional modalities (e.g., BI-RADS 2 or 5) will not benefit from receptor imaging. Consequently, we focused on patients with BI-RADS 4 masses, which require further assessment to improve disease management.

In recent decades, a series of molecular imaging probes targeting a single receptor, such as ${ }^{99 \mathrm{~m}} \mathrm{Tc}$-bombesin and ${ }^{99 \mathrm{~m}} \mathrm{Tc}-$ (HYNIC-3PRGD 2 )(tricine), has been thoroughly investigated for breast tumors (19). Nevertheless, the clinical utility of these probes may be restricted by several factors, such as receptor density, binding affinity, and in vivo pharmacokinetics (13). Moreover, surface receptors on tumor cells are always heterogeneous and inhomogeneous $(16,22)$. For example, GRPR is overexpressed in $70 \%$ of breast cancer cells, and integrin $\alpha_{v} \beta_{3}$ is overexpressed in $58 \%$ of breast cancer cells. Breast cancer can also be categorized as estrogen-dependent or estrogen-independent on the basis of presence or absence of estrogen receptors, respectively (23). The estrogen-dependent category exhibits high GRPR expression but low to moderate integrin $\alpha_{\mathrm{v}} \beta_{3}$ expression. In contrast, estrogen-independent disease expresses high levels of integrin $\alpha_{v} \beta_{3}$ but has undetectable GRPR expression. Therefore, we selected the dual-targeted ${ }^{99 \mathrm{~m}} \mathrm{Tc}$-RGD-bombesin probe in this study to increase detection efficacy.

Indeed, ${ }^{99} \mathrm{~m}$ Tc-RGD-bombesin SPECT showed good efficacy in the detection of breast cancer. Specifically, the qualitative and semiquantitative analyses clearly detected 20 malignant masses,

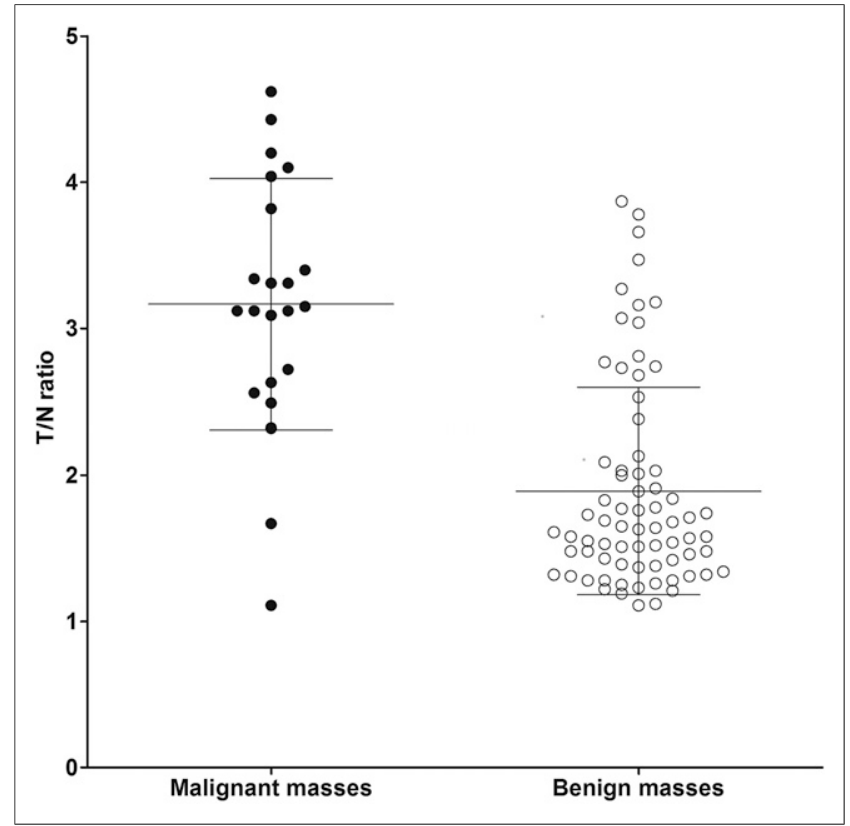

FIGURE 3. T/Ns of ${ }^{99 m}$ Tc-RGD-bombesin in benign and malignant masses $(P<0.05)$. 


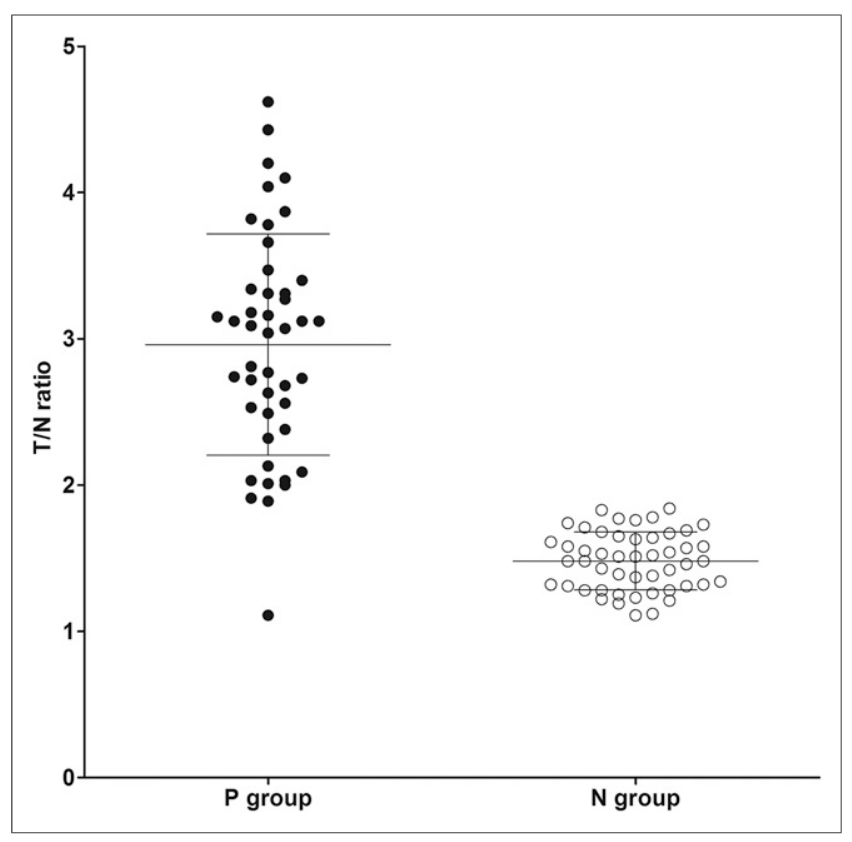

FIGURE 4. T/Ns of ${ }^{99 m T C-R G D-b o m b e s i n ~ i n ~ t h e ~} 2$ groups $(P<0.05)$.

for a sensitivity of $90.9 \%$, which was higher than that of RGD or bombesin monomeric radiotracers (19). We speculate that the high sensitivity may be attributable to the unique advantages of dualtargeted probes. Of the 22 malignant masses in this study, 10 exhibited a coexpression pattern $\left(\mathrm{GRPR}^{+} / \alpha_{\mathrm{v}} \beta_{3}{ }^{+}\right), 11$ a single expression pattern ( $5 \mathrm{GRPR}^{-} / \alpha_{\mathrm{v}} \beta_{3}{ }^{+}$and $6 \mathrm{GRPR}^{+} / \alpha_{\mathrm{v}} \beta_{3}{ }^{-}$), and 1 a dual-negative expression pattern $\left(\mathrm{GRPR}^{-} / \alpha_{\mathrm{v}} \beta_{3}{ }^{-}\right)$. These results indicate that as a dual-targeted probe, ${ }^{99 \mathrm{~m} T c-R G D-b o m b e s i n}$ SPECT is effective even in breast cancer that expresses only one of the two receptors, resulting in increased sensitivity.

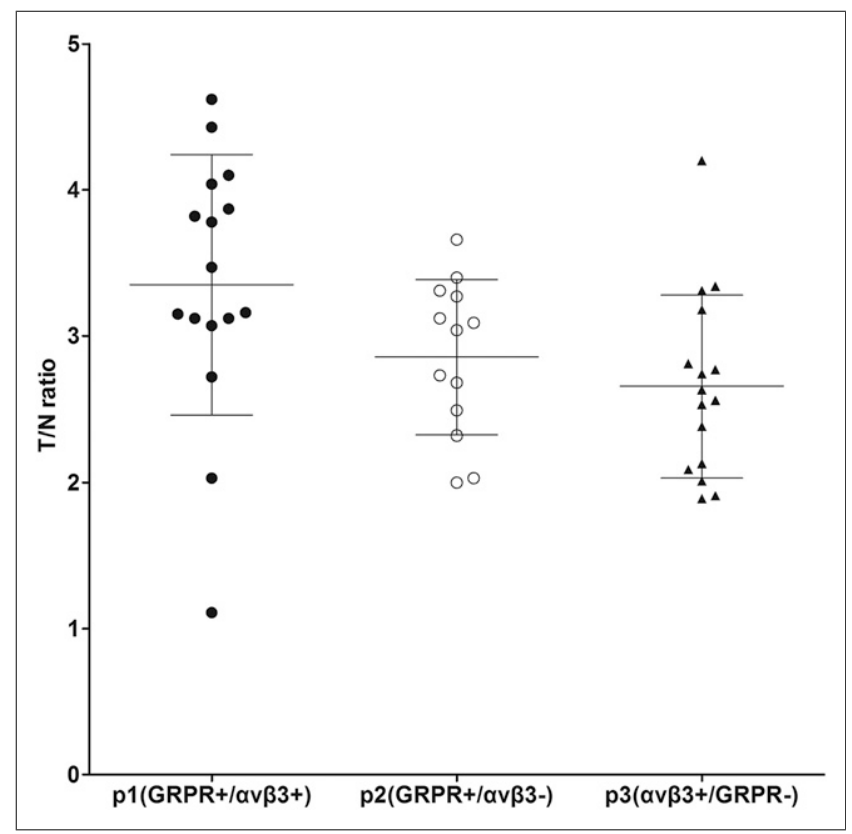

FIGURE 5. T/Ns of $99 \mathrm{mTC}$-RGD-bombesin in the 3 subgroups $(P>0.05)$.
A perfectly organized diagnostic procedure is crucial for patients. Our primary aim was to develop a method to reduce the number of unnecessary biopsies. As demonstrated by our results, ${ }^{99 m}$ Tc-RGDbombesin SPECT can identify benign masses on the basis of a lack of ${ }^{99 \mathrm{~m} T c-R G D-b o m b e s i n}$ uptake and low T/Ns. Our investigation revealed that the negative predictive values of the qualitative and semiquantitative analyses were $96.0 \%$ and $96.6 \%$, respectively. Therefore, at least $51.1 \%$ of these recruited patients can avoid the anxiety associated with biopsy and instead elect to undergo follow-up examinations. In other words, ${ }^{99 \mathrm{~m}} \mathrm{Tc}-\mathrm{RGD}$-bombesin SPECT can decrease the biopsy rate and improve patient safety with a high negative predictive value. These gratifying imaging results may allow ${ }^{99 m}$ Tc-RGD-bombesin SPECT to be extended to BI-RADS 4 masses to avoid unnecessary biopsy.

There were 2 false-negative results in this study. One was a mass smaller than $1 \mathrm{~cm}(0.6 \mathrm{~cm})$ in the lower inner quadrant of the breast, which is distant from the SPECT detector. The other was a mass double-negative for GRPR and $\alpha_{\mathrm{v}} \beta_{3}$ expression $\left(\mathrm{GRPR}^{-} / \alpha_{\mathrm{v}} \beta_{3}{ }^{-}\right.$), thus showing no ${ }^{99 \mathrm{~m} T c-R G D-b o m b e s i n}$ uptake on the SPECT images. Previous studies confirmed that the utility of nuclear receptor imaging may be limited for masses that are small, deep, or of a rare receptor expression pattern $(19,24)$.

False-positive cases occurred at a much higher rate, possibly because of the nonspecific expression of GRPR and integrin $\alpha_{v} \beta_{3}$. It is well known that integrin $\alpha_{v} \beta_{3}$ receptor is highly expressed not only in tumor vasculature and various tumor cells but also in other related pathologic processes (e.g., inflammation, rheumatism, and tuberculosis) $(19,24,25)$. Similarly, GRPR overexpression can also be found in benign breast tissues (24). Our immunohistochemistry results also verified that at least one receptor (GRPR or integrin $\alpha_{v} \beta_{3}$ ) was expressed in all false-positive cases, directly resulting in relatively high uptake of ${ }^{99 m}$ Tc-RGD-bombesin and the low specificity observed.

We analyzed the $99 \mathrm{~m}$ Tc-RGD-bombesin images both qualitatively and semiquantitatively, and both methods had the same sensitivity. Among the 24 cases that were false-positive on qualitative analysis, the possibility of malignancy was excluded in 8 cases because of their relatively low $\mathrm{T} / \mathrm{N}$ on semiquantitative analysis. This finding implies that qualitative analysis alone may result in bias because it is subjective, although the final specificity of the two analysis methods did not significantly differ. Therefore, we suggest that they should be combined.

This study had several limitations. First, an analytic precondition is a sufficient number of samples. Only one case of malignancy had

TABLE 2

Efficacy of ${ }^{99 m}$ Tc-RGD-Bombesin SPECT Using Qualitative and Semiquantitative Analyses

\begin{tabular}{|c|c|c|c|}
\hline Index (\%) & $\begin{array}{l}\text { Qualitative } \\
\text { analysis }\end{array}$ & $\begin{array}{c}\text { Semiquantitative } \\
\text { analysis }\end{array}$ & $P$ \\
\hline Sensitivity & 90.9 & 90.9 & $>0.05$ \\
\hline Specificity & 66.7 & 77.8 & $>0.05$ \\
\hline PPV & 45.5 & 55.6 & $>0.05$ \\
\hline NPV & 96 & 96.6 & $>0.05$ \\
\hline Accuracy & 72.3 & 80.9 & $>0.05$ \\
\hline
\end{tabular}




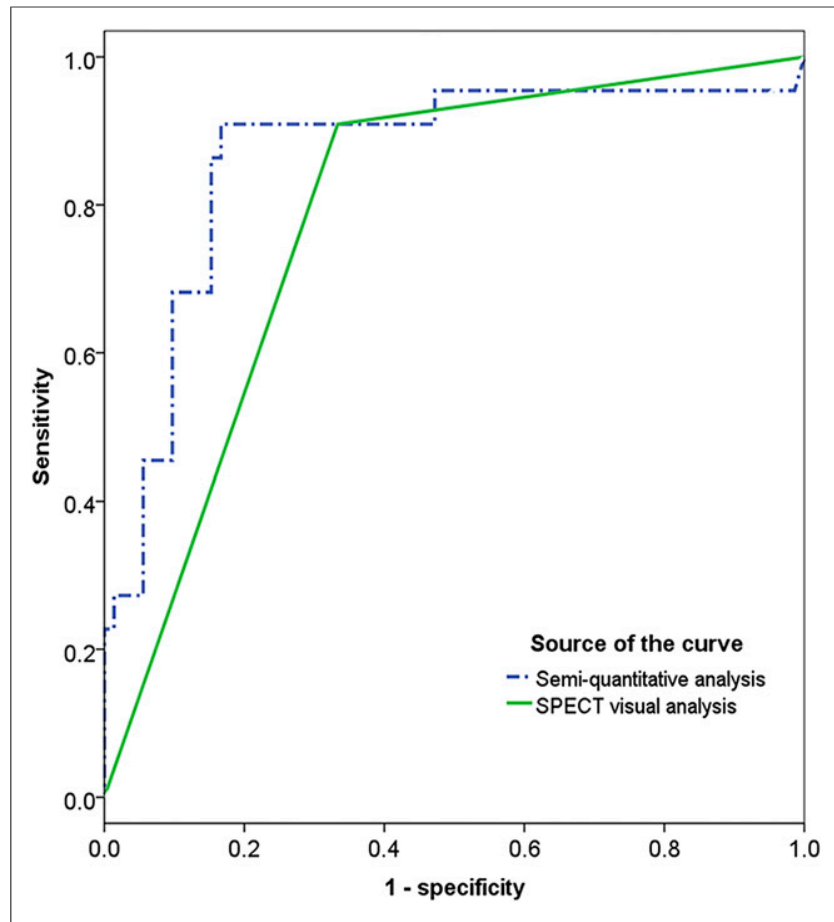

FIGURE 6. Receiver-operating-characteristic curves of SPECT qualitative and semiquantitative analyses.

double-negative receptor expression $\left(\mathrm{GRPR}^{-} / \alpha_{\mathrm{v}} \beta_{3}{ }^{-}\right)$in this study, possibly because the population was relatively restricted (only patients with BI-RADS 4 masses). Since such a small sample cannot determine the exact proportion of this type of cancer, further investigation is warranted. Second, because of the heterodimeric nature of ${ }^{99 m}$ Tc-RGD-bombesin, the exact manner by which this tracer binds to GRPR and $\alpha_{v} \beta_{3}$ receptor is unclear. It has been speculated that high tumor uptake of this tracer results from the increased local concentration, similar to some homodimeric tracers (16). Although highly sensitive, the tracer uptake did not correlate with receptor expression.

\section{CONCLUSION}

${ }^{99 m}$ Tc-RGD-bombesin SPECT showed a high negative predictive value and may provide additional diagnostic information on BI-RADS 4 breast masses. Consequently, patients with SPECTnegative BI-RADS 4 masses may choose careful follow-up rather than immediate biopsy. The findings of this preliminary study need further substantiation using a larger patient population.

\section{DISCLOSURE}

The costs of publication of this article were defrayed in part by the payment of page charges. Therefore, and solely to indicate this fact, this article is hereby marked "advertisement" in accordance with 18 USC section 1734 . This research was supported by grants 81271606 , 81571708, and 81501506 from the National Natural Science Foundation of China (NSFC), grant 20150520154JH from the Research Fund of the Science and Technology Department of Jilin Province, grant
2015Q020 from the Foundation of the National Health and Family Planning Commission of Jilin Province, and grant SCZSY201508 from the Hygiene Specific Subjects of Jilin Province. No other potential conflict of interest relevant to this article was reported.

\section{REFERENCES}

1. DeSantis C, Siegel R, Bandi P, Jemal A. Breast cancer statistics, 2011. CA Cancer J Clin. 2011;61:409-418.

2. Cancer Facts \& Figures 2014. Atlanta, GA: American Cancer Society; 2014.

3. Siegel RL, Miller KD, Jemal A. Cancer statistics, 2015. CA Cancer J Clin. 2015;65:5-29.

4. ACR BI-RADS Atlas. 5th ed. Reston, VA: American College of Radiology; 2013.

5. Mercado CL. BI-RADS update. Radiol Clin North Am. 2014;52:481-487.

6. Bent CK, Bassett LW, D'Orsi CJ, Sayre JW. The positive predictive value of BIRADS microcalcification descriptors and final assessment categories. AJR. 2010;194:1378-1383.

7. Kerlikowske K, Hubbard RA, Miglioretti DL, et al. Comparative effectiveness of digital versus film-screen mammography in community practice in the United States: a cohort study. Ann Intern Med. 2011;155:493-502.

8. Weaver DL, Rosenberg RD, Barlow WE, et al. Pathologic findings from the Breast Cancer Surveillance Consortium: population-based outcomes in women undergoing biopsy after screening mammography. Cancer. 2006;106:732-742.

9. Esserman L, Cowley H, Eberle C, et al. Improving the accuracy of mammography: volume and outcome relationships. J Natl Cancer Inst. 2002;94:369375 .

10. Brewer NT, Salz T, Lillie SE. Systematic review: the long-term effects of falsepositive mammograms. Ann Intern Med. 2007;146:502-510.

11. Yazici B, Sever AR, Mills P, Fish D, Jones SE, Jones PA. Scar formation after stereotactic vacuum-assisted core biopsy of benign breast lesions. Clin Radiol. 2006;61:619-624.

12. Zagouri F, Sergentanis TN, Gounaris A, et al. Pain in different methods of breast biopsy: emphasis on vacuum-assisted breast biopsy. Breast. 2008;17:71-75.

13. Liu Z, Wang F. Dual-targeted molecular probes for cancer imaging. Curr Pharm Biotechnol. 2010;11:610-619.

14. Chen Q, Ma Q, Chen M, et al. An exploratory study on ${ }^{99 m}$ Tc-RGD-BBN peptide scintimammography in the assessment of breast malignant lesions compared to ${ }^{99 \mathrm{~m}} \mathrm{Tc}-3 \mathrm{P}_{4}-\mathrm{RGD}_{2}$. PLoS One. 2015;10:e0123401.

15. Liu Z, Huang J, Dong C, et al. ${ }^{99 m}$ Tc-labeled RGD-BBN peptide for smallanimal SPECT/CT of lung carcinoma. Mol Pharm. 2012;9:1409-1417.

16. Liu Z, Niu G, Wang F, Chen X. ${ }^{68}$ Ga-labeled NOTA-RGD-BBN peptide for dual integrin and GRPR-targeted tumor imaging. Eur J Nucl Med Mol Imaging. 2009;36:1483-1494.

17. Ma Q, Ji B, Jia B, et al. Differential diagnosis of solitary pulmonary nodules using ${ }^{99 m}$ Tc-3P 4 -RGD 2 scintigraphy. Eur J Nucl Med Mol Imaging. 2011;38:2145-2152.

18. Scott N, Millward E, Cartwright EJ, Preston SR, Coletta PL. Gastrin releasing peptide and gastrin releasing peptide receptor expression in gastrointestinal carcinoid tumours. J Clin Pathol. 2004;57:189-192.

19. Liu L, Song Y, Gao S, et al. ${ }^{99 m} \mathrm{Tc}-3 \mathrm{PRGD}_{2}$ scintimammography in palpable and nonpalpable breast lesions. Mol Imaging. 2014;13.

20. Liu Z, Yan Y, Liu S, Wang F, Chen $\mathrm{X} .{ }^{18} \mathrm{~F},{ }^{64} \mathrm{Cu}$, and ${ }^{68} \mathrm{Ga}$ labeled RGD-bombesin heterodimeric peptides for PET imaging of breast cancer. Bioconjug Chem. 2009;20:1016-1025.

21. Okarvi SM. Peptide-based radiopharmaceuticals: future tools for diagnostic imaging of cancers and other diseases. Med Res Rev. 2004;24:357-397.

22. Liu Z, Yan Y, Chin FT, Wang F, Chen X. Dual integrin and gastrin-releasing peptide receptor targeted tumor imaging using ${ }^{18} \mathrm{~F}$-labeled PEGylated RGDbombesin heterodimer ${ }^{18}$ F-FB-PEG3-Glu-RGD-BBN. J Med Chem. 2009;52: $425-432$.

23. Strande V, Canelle L, Tastet C, Burlet-Schiltz O, Monsarrat B, Hondermarck H. The proteome of the human breast cancer cell line MDA-MB-231: analysis by LTQ-Orbitrap mass spectrometry. Proteomics Clin Appl. 2009;3:41-50.

24. Shariati F, Aryana K, Fattahi A, et al. Diagnostic value of ${ }^{99 \mathrm{~m}} \mathrm{Tc}$-bombesin scintigraphy for differentiation of malignant from benign breast lesions. Nucl Med Commun. 2014;35:620-625.

25. Mousa SA. $\alpha \mathrm{v}$ vitronectin receptors in vascular-mediated disorders. Med Res Rev. 2003;23:190-199. 